\title{
A simple and efficient seamless DNA cloning method using SLiCE from Escherichia coli laboratory strains and its application to SLiP site-directed mutagenesis
}

\author{
Ken Motohashi
}

\begin{abstract}
Background: Seamless ligation cloning extract (SLiCE) is a simple and efficient method for DNA assembly that uses cell extracts from the Escherichia coli PPY strain, which expresses the components of the $\lambda$ prophage Red/ET recombination system. This method facilitates restriction endonuclease cleavage site-free DNA cloning by performing recombination between short stretches of homologous DNA ( $\geq 15$ base pairs).

Results: To extend the versatility of this system, I examined whether, in addition to bacterial extracts from the PPY strain, other E. coli laboratory strains were suitable for the SLiCE protocol. Indeed, carefully prepared cell extracts from several strains exhibited sufficient cloning activity for seamless gene incorporation into vectors with short homology lengths (approximately 15-20 bp). Furthermore, SLiCE was applied to the polymerase chain reaction (PCR)-based site-directed mutagenesis method, in a process termed "SLiCE-mediated PCR-based site-directed mutagenesis (SLiP site-directed mutagenesis)". SLiP site-directed mutagenesis simplifies the steps of PCR-based site-directed mutagenesis, as it exploits the capability of the SLiCE method to insert multiple fragments.

Conclusions: SLiCE can be performed in the laboratory with no requirement for a special E. coli strain, and the technique is easily established. This method increases the cloning efficiency, shortens the time for DNA manipulation, and greatly reduces the cost of seamless DNA cloning.
\end{abstract}

Keywords: Homologous recombination, Seamless DNA cloning, SLiCE, Site-directed mutagenesis, Plant redox-related gene

\section{Background}

The manipulation of recombinant DNA molecules is an indispensable step in current molecular biology research. Type IIP restriction endonucleases and DNA ligases were the original "workhorses" utilized to generate plasmids or other types of DNA vectors [1]. Recently, various restriction endonuclease cleavage site-independent cloning methods, which overcome the limitations associated with the lack of unique restriction enzyme sites, have been described [2-13]. These methods are based on homologous recombination, in which overlapping sequences present at the $5^{\prime}$ and 3' ends of DNA fragments are combined.

\section{Correspondence: motohas@cc.kyoto-su.ac.jp}

Department of Bioresource and Environmental Sciences, Faculty of Life Sciences, Kyoto Sangyo University, Kamigamo Motoyama, Kita-ku, Kyoto 603-8555, Japan
Seamless Ligation Cloning Extract (SLiCE), which was initially developed as a novel bacterial cell extract-based DNA cloning method, utilizes the in vitro homologous recombination activity of Escherichia coli cell extracts. Zhang et al. reported that cell extracts from the E. coli PPY strain, which contains the $\lambda$ prophage Red/ET recombination system, could efficiently assemble DNA fragments with short end overlaps of at least 15 base pairs (bp) in vitro [14]. SLiCE is a simple and highly cost-effective approach for vector construction because, apart from adenosine triphosphate (ATP) and commonly used buffers, only an $E$. coli lysate was required for the seamless ligation of DNA fragments into vectors. However, cell extracts from DH10B (a common E. coli laboratory strain) did not exhibit efficient cloning activity 
with short end homology fragments (lengths with a range of 15-20 bp) [14].

Many methods for site-directed mutagenesis have been developed, including QuickChange site-directed mutagenesis [15], the overlap extension method [16, 17], and the megaprimer method [18, 19]. QuickChange site-directed mutagenesis is widely used as a simple polymerase chain reaction (PCR)-based method that does not require the purification of PCR fragments [20-23]. However, mutations may occur in the vector at non-desired sites during PCR amplification, which can compromise the fidelity of the approach. On the other hand, because the overlap extension and megaprimer methods utilize vectors that have been digested with restriction endonucleases, introduction of mutations in the vector region is avoided [24-26]. However, vector construction by these methods requires two sequential PCR reactions, and the purification of insert DNA fragments. To simplify these multiple-step methods, SLiCE has been applied to overlap extension PCR-based site-directed mutagenesis, because it can simultaneously integrate several DNA fragments into a vector [14]. In this study, I demonstrated that SLiCE-mediated PCR-based site-directed mutagenesis (SLiP site-directed mutagenesis) could be performed using extracts of a regular laboratory E. coli strain. This approach can be adopted in studies that require precise generation of mutants in the absence of unwanted alterations to the vector backbone.

\section{Results}

\section{Estimation of SLICE from $E$. coli laboratory strains}

Both the colony formation rate (number of colonies) and the ratio of correct clones (cloning efficiency) in transformation are important determinants for efficient cloning of PCR fragments. Cell lysates from E. coli $\mathrm{RecA}^{-}$strains such as DH10B contain endogenous in vitro homologous recombination activity, and can be used to clone PCR fragments into vectors with homology regions. However, cloning with lysates from this strain is not efficient, particularly in the case of inserts with short homology lengths (approximately 15-20 bp), because of a lower colony formation rate [14]. An E. coli PPY strain that expresses an optimized $\lambda$ prophage Red/ET recombination system circumvents this problem by increasing the colony formation rate during PCR fragment cloning [14]. To extend the utility of this method, I prepared SLiCE extracts from several E. coli laboratory strains with some modifications, and estimated the efficiency with which redox-related genes from Arabidopsis could then be cloned into expression vectors [27-30] (Fig. 1a). The SLiCE extracts from common $\operatorname{RecA}^{-} E$. coli laboratory strains such as DH10B, JM109, DH5 $\alpha$, XL10-Gold and Mach1 T1 supported the cloning of PCR fragments containing a 19-bp overlap region into the vectors, and were associated with a high

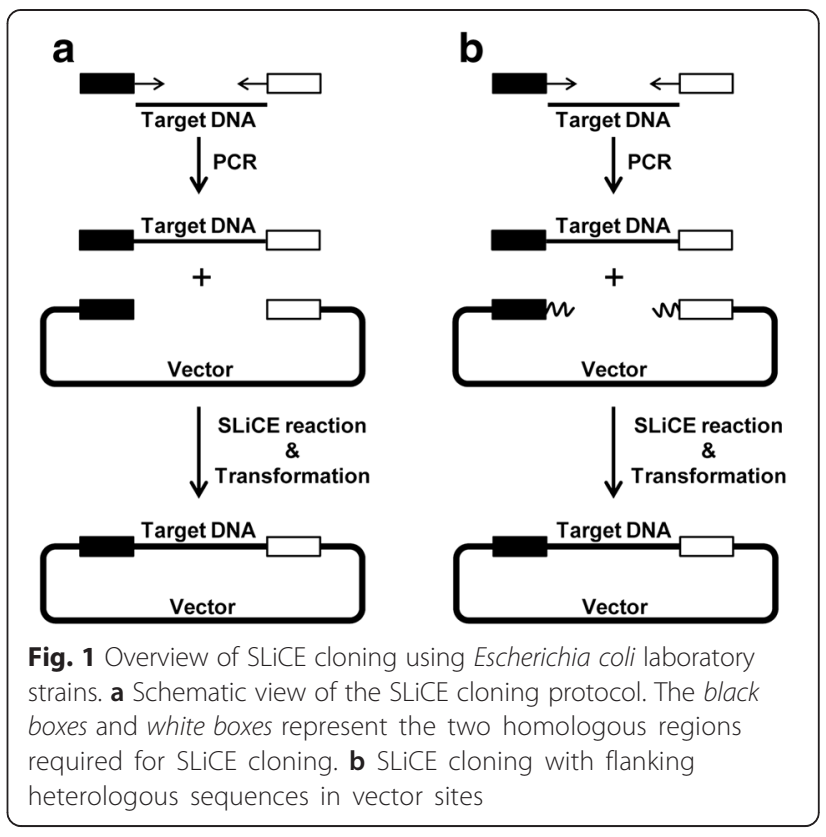

colony formation rate (Table 1). Additionally, SURE2, a $\mathrm{Rec}^{+}$, recB recJ strain, also facilitated the cloning of PCR fragments with almost the same efficiency. No extract that contained only insert DNA fragments and linearized vector could also clone the PCR fragments into vectors using homologous recombination activity in vivo in host cells (Table 1) [31]. However, the transformation efficiencies ("Number of colonies" in Table 1) were less effective with $1 / 40-1 / 200$ of bacterial SLiCE from several E. coli laboratory strains. Together, these data highlight the specificity of the new method. The improved SLiCE preparation could support the cloning of the PCR fragments into vector, with an efficiency of $2-10 \times 10^{3}$ colonies/ng vector; this yield was obtained with a 19-bp overlap region and using $\sim 2 \times 10^{8} \mathrm{CFU} / \mu \mathrm{g}$ of pUC19 DNA chemically competent cells. In contrast, a previously reported SLiCE from the DH10B strain yielded a more modest efficiency of 75-80 colonies/ng vector; in this case, $15-20$ bp overlap lengths and $1 \times 10^{10} \mathrm{CFU} / \mu \mathrm{g}$ of pUC19 DNA high efficiency electrocompetent cells were used [14]. The SLiCEs from E. coli laboratory $\mathrm{Rec}^{-}$strains used in this study were sufficiently active to achieve seamless cloning with respect to both colony formation rate (number of colonies) and the ratio of the correct clones (cloning efficiency). These results indicate that $E$. coli laboratory strains other than $E$. coli PPY can be used as the SLiCE sources. Therefore, SLiCE from the JM109 strain was used in all subsequent experiments.

Next, the effect of short homology length on cloning efficiency was evaluated using PCR fragments containing overlaps of varied lengths (Table 2). To determine whether the cloning fidelity was maintained at all overlap lengths, DNA sequencing was performed. This revealed 
Table 1 The cloning efficiencies using SLiCE from different E. coli laboratory strains

\begin{tabular}{lllll}
\hline Strain & \multicolumn{2}{c}{ Number of colonies $^{\mathrm{a}}$} & \multicolumn{2}{c}{ Cloning efficiency $^{\mathrm{b}}$} \\
\cline { 2 - 5 } & Prx IIE (AT3G52960) & G6PDH1 (AT5G35790) & & Prx IIE (AT3G52960) \\
\hline no extract & $47.0 \pm 4.6$ & $63.7 \pm 5.5$ & $16 / 18$ & $17 / 18$ \\
DH10B & $4,630 \pm 879$ & $3,020 \pm 63.5$ & $17 / 18$ & $18 / 18$ \\
JM109 & $9,960 \pm 240$ & $5,300 \pm 820$ & $18 / 18$ & $18 / 18$ \\
DH5a & $6,130 \pm 348$ & $4,340 \pm 979$ & $18 / 18$ & $18 / 18$ \\
XL10-Gold & $6,210 \pm 652$ & $3,610 \pm 287$ & $17 / 18$ & $18 / 18$ \\
Mach1 T1 & $9,530 \pm 411$ & $2,310 \pm 416$ & $18 / 18$ & $18 / 18$ \\
SURE2 & $8,490 \pm 896$ & $6,040 \pm 1,380$ & $16 / 18$ & $17 / 18$ \\
\hline
\end{tabular}

${ }^{a}$ Number of colonies is represented as CFU per nanogram of vector. Each value of "number of colonies" is the mean \pm standard deviation of three independent experiments. ${ }^{b}$ Cloning efficiencies for the insert DNA are represented as "number of clones with the confirmed correct insert length by colony-PCR/number of colonies subjected to colony-PCR". The insert DNA fragments were amplified using 19-bp overlap primers. The linearized vector DNA was prepared by PCR. The SLiCE reaction was performed for $60 \mathrm{~min}$ at $37^{\circ} \mathrm{C}$ with an insert:vector ratio of 1:1 and 3:1 for Prx IIE and G6PDH1, respectively

that a minimum overlap length of 15 bp was required for correct insertion of the DNA fragments into the vector. Although DNA assembly by homologous recombination with a SLiCE from the PPY strain required more than $15 \mathrm{bp}$ of overlapping homology [14], I found that a 10-bp overlap was sufficient for the assembly of insert DNA into the vector. However, both the cloning efficiency and fidelity of a 10-bp overlap assembly were slightly reduced. PCR fragments with a 19-bp overlap region resulted in the maximum number of colonies upon transformation.

\section{SLiCE can assemble insert DNA fragments into restriction enzyme-digested vectors with flanking heterologous sequences}

PCR-amplified insert DNAs have often been cloned into vectors digested at restriction enzyme cleavage sites as a standard protocol [32-37]. To evaluate the cloning efficiency using such an approach, pET23 vectors were digested with restriction enzymes to yield flanking heterologous sequences (Fig. 1b). The cloning efficiency for the digested vector was determined using multiple pairwise combinations of cloning sites (Table 3). I found that inserts could be incorporated into vectors with heterologous flanking regions, although the cloning efficiency varied according to the precise combination of restriction enzymes used (Table 3 and Additional file 1: Figure S1). The presence of the heterologous flanking region at the $5^{\prime}$ or $3^{\prime}$ end of the vector DNA did not inhibit the correct ligation of the insert DNA to the vector (Table 3; NdeI[or NcoI]-BamHI and BamHI-XhoI). However, the presence of heterologous flanking regions at both the $5^{\prime}$ and 3 ' ends of the vector DNA markedly reduced the cloning efficiency (Table $3 ; \mathrm{BamHI}$ ). The fidelity of colony-PCR positive clones was minimally affected by the presence of the heterologous flanking sequences.

\section{Optimization of the SLiCE reaction}

Next, the conditions for the SLiCE reaction were optimized for extracts from E. coli JM109. Time-course experiments indicated that transformation efficiencies were more than $3 \times 10^{3}$ colonies/ng vector over incubation periods between 5 and $60 \mathrm{~min}$, and the reaction reached saturation rapidly (Fig. 2a). These transformation efficiencies were sufficient for usual DNA cloning, using conventional chemically competent cells. Incubations lasting more than 90 min reduced the colony formation rate. Longer incubation times might induce degradation of the insert and vector DNA, since the SLiCE contains

Table 2 Effect of end homology length on SLiCE cloning

\begin{tabular}{|c|c|c|c|c|c|c|}
\hline \multirow{2}{*}{$\begin{array}{l}\text { Homology } \\
\text { length (bp) }\end{array}$} & \multicolumn{2}{|c|}{ Number of colonies ${ }^{a}$} & \multicolumn{2}{|c|}{ Cloning efficiency } & \multicolumn{2}{|c|}{ Cloning accuracy $(\%)^{b}$} \\
\hline & Prx IE & G6PDH1 & Prx IE & G6PDH1 & Prx IIE & G6PDH1 \\
\hline 10 & $558 \pm 74.5$ & $585 \pm 121$ & $11 / 16$ & $16 / 16$ & 100.0 & 93.8 \\
\hline 15 & $875 \pm 43.9$ & $777 \pm 186$ & $16 / 16$ & $15 / 16$ & 100.0 & 100.0 \\
\hline 19 & $972 \pm 162$ & $1,070 \pm 289$ & $15 / 16$ & $16 / 16$ & 100.0 & 100.0 \\
\hline 24 & $926 \pm 28.6$ & $519 \pm 74.8$ & $16 / 16$ & $16 / 16$ & 100.0 & 100.0 \\
\hline 29 & $483 \pm 34.8$ & $520 \pm 51.4$ & $16 / 16$ & $16 / 16$ & 100.0 & 100.0 \\
\hline 34 & $105 \pm 37.2$ & $150 \pm 47.1$ & $14 / 16$ & $16 / 16$ & 100.0 & 87.5 \\
\hline
\end{tabular}

aEach value of "number of colonies" is the mean \pm standard deviation of three independent experiments. ${ }^{\mathrm{b}} \mathrm{Cloning}$ accuracies are given as the percentage of correctly cloned expression vectors in colony-PCR positive clones. The insert DNA fragments and linearized vector DNA were prepared by PCR. The SLiCE (JM109) reaction was performed for $10 \mathrm{~min}$ at $37^{\circ} \mathrm{C}$ with 1:1 and 3:1 molar ratios of insert to vector for Prx IIE and G6PDH1, respectively 
Table 3 The SLiCE cloning efficiencies of linearized vectors prepared by digestion with different restriction enzymes

\begin{tabular}{|c|c|c|c|c|c|c|c|}
\hline \multirow{2}{*}{$\begin{array}{l}\text { Restriction } \\
\text { enzymes }\end{array}$} & \multirow{2}{*}{$\begin{array}{l}\text { Flanking heterologous } \\
\text { length (bp) }\end{array}$} & \multicolumn{2}{|c|}{ Number of colonies ${ }^{a}$} & \multicolumn{2}{|c|}{ Cloning efficiency } & \multicolumn{2}{|c|}{ Cloning accuracy (\%) } \\
\hline & & $\operatorname{Prx} \| \mathrm{E}$ & G6PDH1 & Prx IIE & G6PDH1 & Prx IIE & G6PDH1 \\
\hline Ndel - Xhol & $0+0$ & $9,680 \pm 651$ & - & $18 / 18$ & - & 100 & - \\
\hline Ncol - Xhol & $0+0$ & - & $8,470 \pm 2,180$ & - & $17 / 18$ & - & 94.1 \\
\hline BamHI & $40+40$ & $926 \pm 62.0$ & $576 \pm 17.6$ & $1 / 18$ & $5 / 18$ & 100 & 100 \\
\hline Ndel - BamHI & $0+40$ & $164 \pm 20.8$ & - & $12 / 18$ & - & 75.0 & - \\
\hline $\mathrm{Ncol}-\mathrm{BamHI}$ & $0+40$ & - & $195 \pm 83.3$ & - & $4 / 18$ & - & 100 \\
\hline BamHI - Xhol & $40+0$ & $857 \pm 298$ & $960 \pm 71.3$ & $9 / 18$ & $12 / 18$ & 88.9 & 83.3 \\
\hline
\end{tabular}

bacterial nucleases. A 1:1 to 3:1 molar ratio of insert:vector yielded highly efficient cloning, with more than $2 \times 10^{3}$ colonies/ng vector being formed (Fig. 2b). The enhancement of transformation efficiency in $E$. coli by modification of the SLiCE preparation protocol allows the simplification of several steps, including purification of insert and vector DNA fragments. Therefore, the influence of the DNA fragment purification steps on cloning efficiency was also evaluated (Table 4). Unpurified PCR fragments could be inserted to the vector, although with a lower transformation efficiency. By contrast, ethanol precipitation or ExoSAP-IT (Affymetrix, Santa Clara, CA) treatment of insert DNA fragments improved the transformation efficiency between 5- and 7-fold. PCR purification columns or purification via agarose gel electrophoresis improved the efficiency between 37- and 138-fold. We also observed a high cloning efficiency (at the optimal insert:vector ratio) regardless of the purification steps used.

\section{Application of SLiCE to PCR-based site-directed mutagenesis}

Previously, the SLiCE method, using the in vitro homologous recombination activity of lysates from the E. coli PPY strain, was employed to integrate multiple DNA fragments into vectors in a single cloning reaction [14]. The overlap extension method, which is used for PCRbased site-directed mutagenesis (Fig. 3a), has an advantage in that it can use restriction enzyme-digested vectors, which excludes the possibility of acquiring a mutation in the vector region $[16,17]$. SLiCE was applied to generate cysteine-to-serine mutants at critical residues in redoxrelated proteins $[26,38-40]$. To generate the cysteine mutants, pairs of insert DNA fragments containing a mutation site and short end homologies at both sides were amplified by PCR, and two fragments were simultaneously integrated into pET vectors using SLiCE (Fig. 3b). Both unpurified and column-purified DNA fragments could be inserted into the vector. The use of column-purified insert DNA increased the number of colonies following transformation by approximately 4- to 16-fold, although the cloning efficiencies were not improved (Table 5). Thus, this new SLiCE-mediated PCR-based site-directed mutagenesis (SLiP site-directed mutagenesis) technique simplifies the original overlap extension method by removing several steps. Although QuickChange site-directed mutagenesis also minimizes the number of steps, SLiP sitedirected mutagenesis has the added advantage that only the insert region (and not the whole plasmid) is amplified by PCR.

\section{Discussion}

In this study, I demonstrated that a wide variety of laboratory $\mathrm{RecA}^{-}$strain extracts can be used to clone PCR fragments, in the absence of any special modifications to the strains. In the case of short homology lengths (approximately 15-20 bp), SLiCEs from several E. coli laboratory strains yielded good colony formation rates, which were at least three orders of magnitude greater than those obtained in a previous study [14]. Unexpectedly, SURE2, which is a $\operatorname{Rec} \mathrm{A}^{+}$, $r e c \mathrm{~B}$ recJ strain, also retained SLiCE activity. Although the mechanisms associated with the in vitro homologous recombination activity of $E$. coli extracts are not fully characterized, routine cloning using several $E$. coli extracts works well even with cells that are not highly electrocompetent. The SLiCEs from $E$. coli laboratory strains in this study have such high transformation efficiencies because several steps of the original SLiCE preparation protocol [14] have been modified. Firstly, E. coli cells were harvested at $\mathrm{OD}_{600}=2.0-3.0$ (late $\log$ phase). Secondly, extraction of $E$. coli lysates was performed under $4{ }^{\circ} \mathrm{C}$, and ice-cold solutions were used during the preparation. These modifications might improve the transformation efficiency following the use of SLiCEs from $E$. coli laboratory strains.

In addition, the SLiCE method was simplified by optimizing the reaction conditions (Fig. 2 and Table 4). Although purification of insert DNA fragments was not essential for the assembly of fragments into the vectors, selecting a purification step improved cloning efficiency (Table 4). A 19-bp overlap sequence is recommended as 


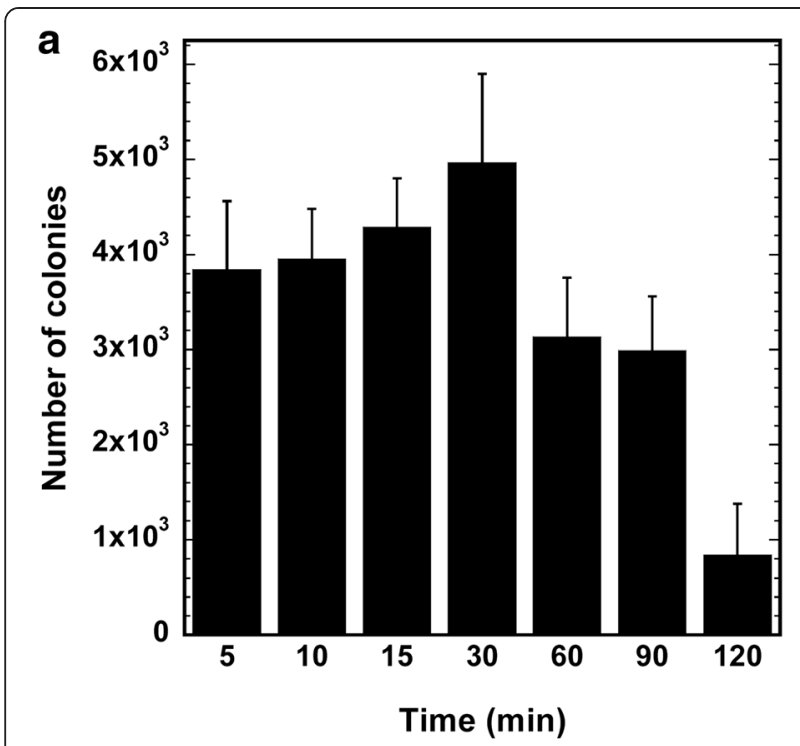

b

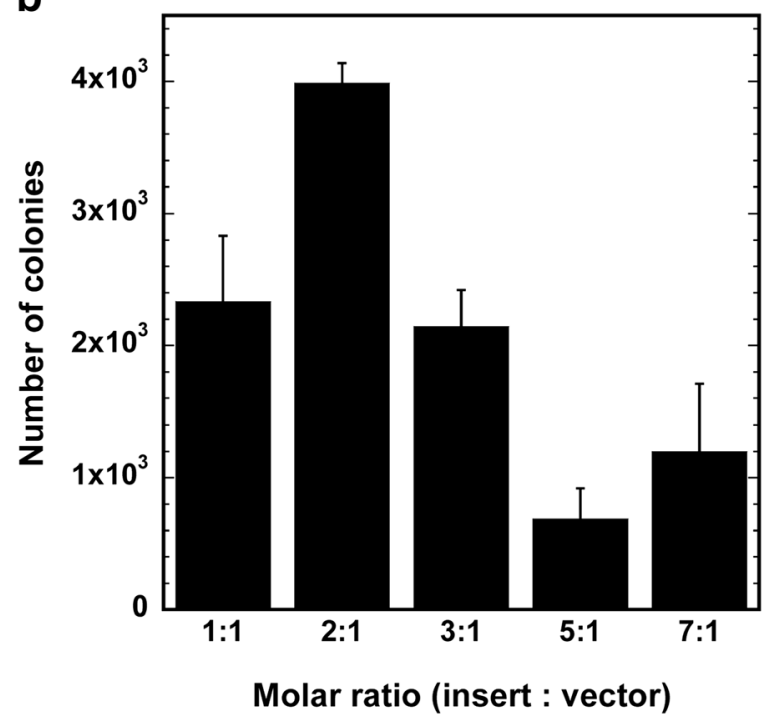

Fig. 2 Optimization of the SLiCE reaction. The insert glucose-6phosphate dehydrogenase 1 (G6PDH1) DNA fragments were amplified using 19-bp overlap primers. The linearized vectors were prepared by PCR amplification. The SLiCE reaction (from JM109 strain of E. coli) was performed at $37{ }^{\circ} \mathrm{C}$. Each value is the mean \pm standard deviation of three independent experiments. a Time course of the SLiCE reaction. The insert G6PDH1 DNA fragments and linearized vector were mixed in a molar ratio of 2:1. $\mathbf{b}$ The effect of the molar ratio of insert (G6PDH1) DNA fragment to vector on the number of colonies formed. The SLiCE reaction was performed for $10 \mathrm{~min}$

the short end homology region, since this yielded the maximum number of transformants (Table 2). However, a 15-bp overlap sequence was also sufficient to provide colonies harboring the correct clone. The SLiCE method also has the advantage that linearized vectors (generated by either PCR amplification or restriction enzyme digestion) may be used, as its efficiency and accuracy were
Table 4 Effect of PCR fragment purification method on SLiCE efficiency

\begin{tabular}{lccccc}
\hline $\begin{array}{l}\text { Purification } \\
\text { method }\end{array}$ & \multicolumn{2}{c}{ Number of colonies $^{f}$} & & \multicolumn{2}{c}{ Cloning efficiency } \\
\cline { 2 - 3 } & Prx IIE & G6PDH1 & & Prx IIE & G6PDH1 \\
\hline None $^{a}$ & $86.3 \pm 2.3$ & $76.0 \pm 4.0$ & & $15 / 18$ & $17 / 18$ \\
EtOH ppt $^{\text {b }}$ & $546 \pm 102$ & $514 \pm 68.7$ & & $18 / 18$ & $17 / 18$ \\
ExoSAP-IT $^{c}$ & $481 \pm 117$ & $507 \pm 105$ & & $18 / 18$ & $18 / 18$ \\
Column $^{d}$ & $7,170 \pm 806$ & $4,660 \pm 1,400$ & & $18 / 18$ & $18 / 18$ \\
Agarose gel $^{\mathrm{e}}$ & $11,900 \pm 1,750$ & $2,830 \pm 100$ & & $18 / 18$ & $18 / 18$ \\
\hline
\end{tabular}

The insert DNA fragments were amplified using 19-bp overlap primers and KOD DNA polymerase [51] (for Prx IIE gene) and PrimeSTAR Max DNA polymerase (for G6PDH1). The linearized vector DNA was prepared by PCR. The PCR solutions were treated with Dpnl. The SLiCE (JM109) reaction was performed for $10 \mathrm{~min}$ at $37^{\circ} \mathrm{C}$. ${ }^{\mathrm{U}}$ Unpurified insert DNA was directly used for the SLiCE reaction. ${ }^{\mathrm{b}}$ Insert DNA was precipitated by ethanol. ${ }^{\mathrm{C}} \mathrm{PCR}$ solution was treated with ExoSAP-IT (Affymetrix). ${ }^{d}$ Insert DNA was purified from the PCR solution using a Gel/PCR

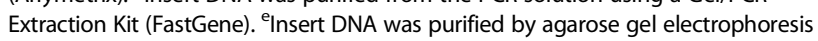
and using a Gel/PCR Extraction Kit (FastGene). An equivalent volume to 1/20 of the PCR solution was used for the SLiCE reaction. "Each value of "number of colonies" is the mean \pm standard deviation of three independent experiments

not affected by the presence of blunt ends or $5^{\prime}$ sequence overhangs at the ends of the vector (Table 2 and Table 3). I summarize the SLiCE protocol from E. coli laboratory strains in Fig. 4. For the standard protocol, various insert DNA fragments, including small amounts of PCR-products amplified as multiple bands can be cloned into vectors with high efficiency. In contrast, a rapid protocol is available for high abundance PCR-products that have been amplified as a single band.

Seamless DNA assembly kits based on in vitro homologous recombination activity have recently become

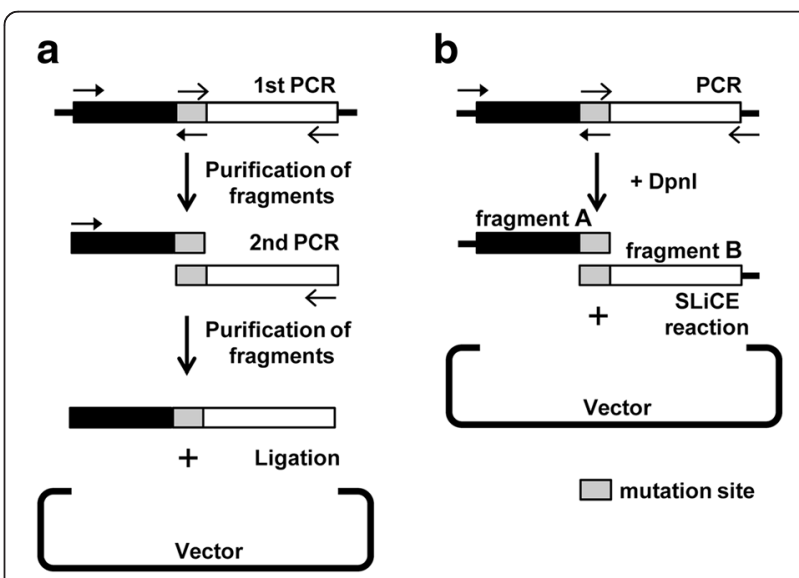

Fig. 3 Outline of PCR-based site-directed mutagenesis using SLiCE. Arrows represent PCR primers. a Original overlap extension method for site-directed mutagenesis. b SLiCE-mediated PCR-based site-directed mutagenesis (SLiP site-directed mutagenesis). The linearized vectors were prepared by PCR amplification or by digesting the vector with restriction enzymes. Dpnl was active in the PCR buffers. In the case of linearized vectors prepared by restriction enzymes, Dpnl added to the insert DNA fragment mixture was heat-inactivated for $15 \mathrm{~min}$ at $80^{\circ} \mathrm{C}$ 
Table 5 SLiCE-mediated PCR-based site-directed mutagenesis

\begin{tabular}{lccc}
\hline Mutation site & Number of colonies $^{c}$ & Cloning efficiency & Mutation(\%) $^{d}$ \\
\hline Prx IIE C51S & $22.0 \pm 14.0$ & $10 / 16$ & 100 \\
Prx IIE C76S & $18.0 \pm 7.5$ & $15 / 16$ & 100 \\
G6PDH1 C975 & $32.0 \pm 4.6$ & $9 / 16$ & 93.3 \\
G6PDH1 C105S & $54.0 \pm 13.5$ & $9 / 16$ & 88.9 \\
Prx IIE C51S & $197 \pm 49.2$ & $14 / 16$ & 100 \\
Prx IIE C76S & $283 \pm 21.2$ & $15 / 16$ & 100 \\
G6PDH1 C975 & $189 \pm 39.0$ & $6 / 16$ & 100 \\
G6PDH1 C105S & $220 \pm 30.0$ & $9 / 16$ & 100 \\
\hline
\end{tabular}

Target cysteine residues that are reduced by chloroplast thioredoxins were substituted with serine residues [26, 52]. The insert DNA fragments were prepared by PCR using PrimeSTAR Max DNA polymerase. pET23a and pET23d vectors were linearized using restriction enzymes Ndel and Xhol (for pET23a) and Ncol and Xhol (for pET23d). The SLiCE (JM109) reaction was performed for $60 \mathrm{~min}$ at $37{ }^{\circ} \mathrm{C}$. ${ }^{\circ}$ One microliter of unpurified insert DNA fragments in $20 \mu \mathrm{L}$ PCR solution was directly used for the SLiCE reaction. ${ }^{b} P C R$ solution $(20 \mu \mathrm{L}$ ) was purified using a Gel/PCR Extraction Kit (FastGene) and an equivalent volume to 1/20 of the PCR solution was used for the SLiCE reaction. "Each value of "number of colonies" is the mean \pm standard deviation of three independent experiments. "The ratio of mutants generated is given as the percentage of mutated clones (determined by DNA sequencing) among colony PCR-positive clones

commercially available. These include the In-Fusion HD Cloning Kit [41, 42] (Clontech), GeneArt Seamless Cloning and Assembly Kit (Life Technologies), and Gibson Assembly Cloning Kit [2, 43] (New England BioLabs). However, many of the commercially available kits are associated with a high cost per reaction. The SLiCE method uses extracts from common E. coli laboratory strains. These extracts can be prepared easily in the laboratory and the use of this method greatly reduces the costs associated with DNA manipulation. SLiCE is highly efficient and maintains high fidelity for gene cloning. These results also allow the use of either commercial or

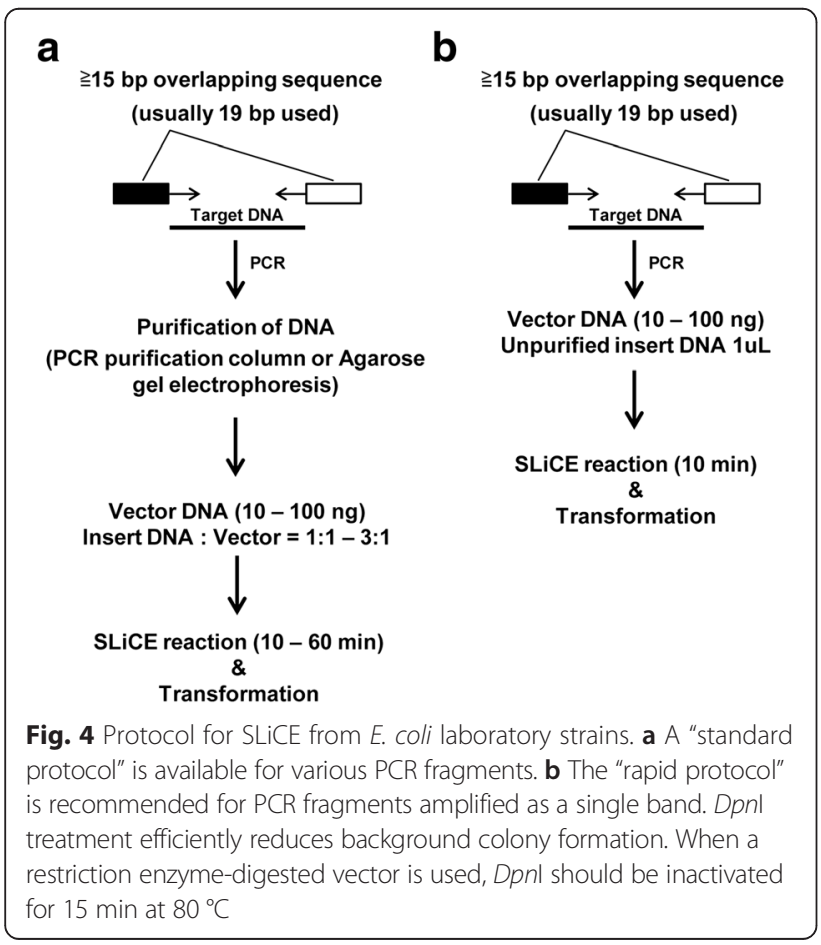

laboratory "homemade" chemically competent cells. As an approximate guide, our laboratory has successfully used SLiCE with chemically competent $E$. coli cells that yield $>10^{7} \mathrm{CFU} / \mu \mathrm{g}$ of pUC19 DNA [44].

PCR-based site-directed mutagenesis is a common technique used in molecular biology experiments. The QuickChange mutagenesis kit is frequently used for sitedirected mutagenesis because it is a simple process that does not require the purification of PCR fragments. However, this method relies on PCR amplification of the entire plasmid, which is accompanied by the risk of introducing a mutation in the vector region. In contrast, in the overlap extension method only the insert DNA is amplified. Furthermore, the possibility of introducing a mutation in the vector region is excluded by the use of a restriction enzyme-digested vector. A limitation of the overlap extension method is that it follows an elaborate protocol and requires multiple steps (Fig. 3a). SLiP sitedirected mutagenesis removes one PCR cycle and two DNA purification steps from the overlap extension method, and thus provides an efficient and accurate method for the generation of site-directed mutants.

\section{Conclusions}

SLiCEs prepared from a wide variety of laboratory $\mathrm{RecA}^{-}$ E. coli strains could be used to clone PCR fragments, in the absence of any special modifications to the strains. SLiP site-directed mutagenesis is a simple and efficient method that removes any possibility of mutation in the vector region. The combination of SLiCE using E. coli laboratory strains and SLiP site-directed mutagenesis will facilitate the generation of recombinant plasmids and mutants, and shorten the time for DNA manipulation in the laboratory. The elucidation of mechanisms that control in vitro homologous recombination activity in $E$. coli lysates will lead to further enhancement of SLiCE efficiency. 


\section{Methods \\ Reagents}

CelLytic B Cell Lysis Reagent (SIGMA B7435) was used for $E$. coli cells lysis buffer. Adenosine- $5^{\prime}$-triphosphate disodium salt hydrate from yeast (Nacalai Tesque, Inc. 01072-24) was used for ATP contained in SLiCE buffer $(10 \times)$.

\section{E. coli strains}

The following E. coli laboratory strains were used to prepare SLiCE extracts (Additional file 1: Table S1): DH10B [45], JM109 [46], DH5 $\alpha$ [47], XL10-Gold (Agilent Technologies, Santa Clara, CA), Mach1 T1 (Life Technologies, Carlsbad, CA) and SURE2 (Agilent Technologies, Santa Clara, CA). ECOS X Competent E. coli DH5a (Nippon gene, Tokyo, Japan) chemically competent cells were used to transform the recombinant DNA generated using the SLiCE method, in order to obtain the constant transformation efficiency.

\section{Preparation of the SLiCE extract from E. coli laboratory strains}

E. coli strains precultured in LB Miller medium $(1 \mathrm{~mL})$ at $37{ }^{\circ} \mathrm{C}$ were transferred to $2 \times$ YT medium $(50 \mathrm{~mL})$ in a 100-mL round-bottom, long-neck Sakaguchi shake flask. The cells were grown at $37{ }^{\circ} \mathrm{C}$ in a reciprocal shaker (160 rpm) until $\mathrm{OD}_{600}$ reached a value of 2.0-3.0 (late log phase). The cultures were generally incubated for 3.5-6.5 h. The cells were harvested by centrifugation at $5,000 \times g$ for $10 \mathrm{~min}$ at $4{ }^{\circ} \mathrm{C}$. The cells were then washed with $50 \mathrm{~mL}$ sterilized water (ice-cold), and centrifuged at $5,000 \times g$ for $5 \mathrm{~min}$ at $4{ }^{\circ} \mathrm{C}$. The wet cells were recovered with a yield of $0.25-0.40 \mathrm{~g}$, and gently resuspended in $1.2 \mathrm{~mL}$ CelLytic B Cell Lysis Reagent and incubated for $10 \mathrm{~min}$ at room temperature. The cell lysates were then centrifuged at $20,000 \times g$ for $2 \mathrm{~min}$ at $4{ }^{\circ} \mathrm{C}$. All subsequent procedures were performed on ice. The supernatants were carefully transferred into $1.5-\mathrm{mL}$ microtubes to remove the insoluble materials, and an equal volume of ice-cold $80 \%$ (v/v) glycerol was added and mixed gently. Forty microliters of each SLiCE extract was aliquoted into a 0.2-mL 8-strip PCR tube. The SLiCE extracts were snapfrozen in a bath of liquid nitrogen, and stored at $-80{ }^{\circ} \mathrm{C}$ in $40 \%$ (v/v, final concentration) glycerol. For short-term storage, the aliquoted SLiCE extracts could be stored at $-20{ }^{\circ} \mathrm{C}$ in $40 \%$ (v/v, final concentration) glycerol for about 3 months without significant loss of activity.

\section{SLiCE reaction and transformation}

SLiCE buffer (10×; 500 mM Tris-HCl, pH 7.5, $100 \mathrm{mM}$ $\mathrm{MgCl}_{2}, 10 \mathrm{mM} \mathrm{ATP}$, and $10 \mathrm{mM}$ dithiothreitol) was passed through a $0.2-\mu \mathrm{m}$ filter, and dispensed in $40-\mu \mathrm{L}$ aliquots into $0.2-\mathrm{mL} 8$-strip PCR tubes and stored at $-20{ }^{\circ} \mathrm{C}$. The Arabidopsis type II peroxiredoxin E (Prx IIE, $0.6 \mathrm{k}$ base pairs (kbp), AT3G52960) [48, 49] and chloroplastic glucose-6-phosphate dehydrogenase 1 (G6PDH1, $1.6 \mathrm{kbp}$, AT5G35790) [50] genes were used as insert DNA molecules. The linearized pET23a DNA and insert DNA fragments were amplified using the primers listed in Additional file 1: Table S2 and Tks Gflex DNA polymerase (Takara-Bio, Otsu, Japan) by PCR. To compare the reaction efficiency of SLiCE with that of restriction enzyme-digested vectors, the pET23a and pET23d vectors were linearized by digestion with restriction enzyme and purified using a Gel/ PCR Extraction Kit (FastGene) following agarose gel electrophoresis.

After the SLiCE reaction solution had been prepared, the SLiCE extract was thawed on ice and mixed gently. The standard SLiCE reaction solution comprised the following components: $10 \mathrm{ng}$ linear vector (PCR amplified or restriction enzyme-digested), an appropriate amount of insert DNA (1:1 to 3:1 molar ratio of insert to vector), $1 \mu \mathrm{L} 10 \times$ SLiCE buffer, $1 \mu \mathrm{L}$ SLiCE extract, and sterilized distilled water to a total volume of $10 \mu \mathrm{L}$. The SLiCE reaction mixture was incubated at $37{ }^{\circ} \mathrm{C}$ for the indicated time (5-120 $\mathrm{min})$. One microliter of the SLiCE reaction solution was chemically transformed into $20 \mu \mathrm{L}$ ECOS X Competent E. coli DH5a (Nippon gene, Tokyo, Japan) according to the instruction manual. The transformation efficiency of the competent cells $(20 \mu \mathrm{L})$ was approximately $2 \times 10^{8} \mathrm{CFU} / \mu \mathrm{g}$ of pUC19 DNA. Transformed $E$. coli cells were plated on LB agar plates containing ampicillin and incubated at $37^{\circ} \mathrm{C}$ for $12-16 \mathrm{~h}$.

\section{Evaluation of the SLiCE reaction}

Various parameters were used to measure the efficiency of the SLiCE reactions under each experimental condition. Number of colonies was represented as CFU per nanogram of vector. Cloning efficiencies for the insert DNA were given as the ratio of colonies with an insert of the confirmed correct length as estimated by colony-PCR. In particular, cloning efficiencies were represented as "the number of clones with the confirmed correct length of insert DNA by colony-PCR/number of colonies subjected to colony-PCR". The cloning accuracy was expressed as the percentage of correctly cloned expression vectors in colony-PCR positive clones. The insert cloning sites were determined by DNA sequencing.

\section{Insert-check by colony PCR in transformed E. coli}

Each colony was picked with a sterile toothpick and put into the bottom of a $0.2-\mathrm{mL} 8$-strip PCR tube or a 96well PCR plate. After the toothpicks were removed from the PCR-tube, $10 \mu \mathrm{L}$ of KAPATaq EXtra DNA polymerase (KAPA Biosystems, Wilmington, MA) PCR mix was added to each sample; this mixture included the T7P and T7T primers corresponding to the T7 promoter and T7 terminator sequences of the $\mathrm{pET}$ vectors, respectively (Additional file 1: Table S3). The sample solutions were 
reacted according to the KAPATaq EXtra standard protocol. When the target DNA was $>1.5 \mathrm{kbp}$ in length, the Tks Gflex DNA polymerase (Takara-Bio, Otsu, Japan) was used instead of KAPATaq EXtra.

\section{SLiCE-mediated PCR-based site-directed mutagenesis (SLiP site-directed mutagenesis)}

Primers for site-directed mutagenesis were designed using the PrimerX program (http://www.bioinformatics. org/primerx/) under the following conditions: melting temperature $>78{ }^{\circ} \mathrm{C}$ and primer termination with guanine $(\mathrm{G})$ or cytidine $(\mathrm{C})$ for QuickChange site-directed mutagenesis kit (Additional file 1: Table S3). Pairs of insert DNA fragments for a cysteine mutant of Prx IIE and G6PDH1 were amplified in a $20-\mu \mathrm{L}$ reaction volume using PrimeSTAR Max DNA polymerase (Takara-Bio, Otsu, Japan) along with the T7P primer/Mutant reverse primers and Mutant forward primers/T7T primer (Additional file 1: Table S3). The two PCR fragments for mutant generation were incubated with $D p n \mathrm{I}$ for $60 \mathrm{~min}$ at $37{ }^{\circ} \mathrm{C}$ to digest the template plasmid DNA. Subsequently, $D p n I$ was inactivated by incubating the mixture at $80{ }^{\circ} \mathrm{C}$ for $15 \mathrm{~min}$. For the unpurified insert DNA fragments, $1 \mu \mathrm{L}$ of each unpurified insert DNA fragment in a 20- $\mu \mathrm{L}$ PCR reaction solution was mixed with the linearized pET23a vector (10 ng; digested using NdeI and XhoI [NcoI and XhoI for pET23d]), and then added to the SLiCE reaction. For the purified insert DNA fragments, an equivalent volume to $1 / 20$ of the PCR solution was added to the SLiCE reaction.

\section{Additional file}

Additional file 1: Figure S1. The multiple cloning site of pET23a (or pET23d) vector. Table S1. Escherichia coli strains used for SLiCE preparation. Table S2. Oligonucleotides for PCR amplification. Table S3. Oligonucleotides for site-directed mutagenesis.

\section{Abbreviations}

ATP: Adenosine triphosphate; CFU: Colony-forming units; G6PDH1: Glucose6-phosphate dehydrogenase 1; PCR: Polymerase chain reaction; Prx IIE: Type II peroxiredoxin E; SLiCE: Seamless ligation cloning extract; SLiP site-directed mutagenesis: SLiCE-mediated PCR-based site-directed mutagenesis.

\section{Competing interests}

The author declares that he has no competing interests.

\section{Authors' contributions}

KM designed and performed the experiments, analyzed the data and wrote the manuscript. The author read and approved the final manuscript.

\section{Acknowledgements}

I thank Yuki Okegawa for critically reading the manuscript. This work was supported by JSPS KAKENHI Grant Numbers 25650037 (to K.M.), and the Science Research Promotion Fund from the Promotion and Mutual Aid Corporation for Private Schools of Japan (to K.M.).

Received: 20 October 2014 Accepted: 5 May 2015

Published online: 03 June 2015

\section{References}

1. Green MR, Sambrook J. Molecular Cloning: A Laboratory Manual, Fourth Edition edn. Cold Spring Harbor: NY Cold Spring Harbor Laboratory Press; 2012.

2. Gibson DG, Young L, Chuang RY, Venter JC, Hutchison 3rd CA, Smith HO Enzymatic assembly of DNA molecules up to several hundred kilobases. Nat Methods. 2009;6(5):343-5.

3. Klock HE, Lesley SA. The Polymerase Incomplete Primer Extension (PIPE) method applied to high-throughput cloning and site-directed mutagenesis. Methods Mol Biol. 2009;498:91-103.

4. Quan J, Tian J. Circular polymerase extension cloning of complex gene libraries and pathways. PLoS One. 2009;4(7):e6441.

5. Li MZ, Elledge SJ. SLIC: a method for sequence- and ligation-independent cloning. Methods Mol Biol. 2012;852:51-9.

6. Warren TD, Coolbaugh MJ, Wood DW. Ligation-independent cloning and self-cleaving intein as a tool for high-throughput protein purification. Protein Expr Purif. 2013;91(2):169-74.

7. Scholz J, Besir H, Strasser C, Suppmann S. A new method to customize protein expression vectors for fast, efficient and background free parallel cloning. BMC Biotechnol. 2013;13:12.

8. Stevenson J, Krycer JR, Phan L, Brown AJ. A practical comparison of ligation-independent cloning techniques. PLoS One. 2013;8(12):e83888.

9. Thieme F, Marillonnet S. Quick and clean cloning. Methods Mol Biol. 2014;1116:37-48.

10. Camilo CM, Polikarpov I. High-throughput cloning, expression and purification of glycoside hydrolases using Ligation-Independent Cloning (LIC). Protein Expr Purif. 2014;99:35-42.

11. Chino A, Watanabe $\mathrm{K}$, Moriya $\mathrm{H}$. Plasmid construction using recombination activity in the fission yeast Schizosaccharomyces pombe. PLoS One. 2010;5(3):e9652.

12. Matsuo Y, Kishimoto H, Horiuchi T, Tanae K, Kawamukai M. Simple and effective gap-repair cloning using short tracts of flanking homology in fission yeast. Biosci Biotechnol Biochem. 2010;74(3):685-9.

13. Goto K, Nagano Y. Ultra-low background DNA cloning system. PLoS One. 2013;8(2):e56530.

14. Zhang Y, Werling U, Edelmann W. SLiCE: a novel bacterial cell extract-based DNA cloning method. Nucleic Acids Res. 2012;40(8):e55.

15. Papworth C, Bauer JC, Braman J, Wright DA. Site-directed mutagenesis in one day with $>80 \%$ efficiency. Strategies. 1996;9:3-4.

16. Higuchi R, Krummel B, Saiki RK. A general method of in vitro preparation and specific mutagenesis of DNA fragments: study of protein and DNA interactions. Nucleic Acids Res. 1988;16(15):7351-67.

17. Ho SN, Hunt HD, Horton RM, Pullen JK, Pease LR. Site-directed mutagenesis by overlap extension using the polymerase chain reaction. Gene. 1989;77(1):51-9.

18. Sarkar G, Sommer SS. The "megaprimer" method of site-directed mutagenesis. Biotechniques. 1990:8(4):404-7.

19. Landt O, Grunert HP, Hahn U. A general method for rapid site-directed mutagenesis using the polymerase chain reaction. Gene. 1990;96(1):125-8.

20. Makyio H, Niwa H, Motohashi K, Taguchi H, Yoshida M. Stabilization of FtsH-unfolded protein complex by binding of ATP and blocking of protease. Biochem Biophys Res Commun. 2002;296(1):8-12.

21. Ueoka-Nakanishi H, Nakanishi Y, Konno H, Motohashi K, Bald D, Hisabori T. Inverse regulation of rotation of F1-ATPase by the mutation at the regulatory region on the gamma subunit of chloroplast ATP synthase. J Biol Chem. 2004;279(16):16272-7

22. Wakita M, Masuda S, Motohashi K, Hisabori T, Ohta H, Takamiya K. The significance of type II and PrxQ peroxiredoxins for antioxidative stress response in the purple bacterium Rhodobacter sphaeroides. J Biol Chem. 2007;282(38):27792-801.

23. Kojima K, Motohashi K, Morota T, Oshita M, Hisabori T, Hayashi H, et al. Regulation of translation by the redox state of elongation factor $\mathrm{G}$ in the cyanobacterium Synechocystis sp. PCC 6803. J Biol Chem. 2009;284(28):18685-91.

24. Roy I, Smith O, Clouthier CM, Keillor JW. Expression, purification and kinetic characterisation of human tissue transglutaminase. Protein Expr Purif. 2013;87(1):41-6.

25. Plattner S, Gruber C, Altmann F, Bohlmann H. Self-processing of a barley subtilase expressed in E. coli. Protein Expr Purif. 2014;101:76-83.

26. Motohashi K, Koyama F, Nakanishi Y, Ueoka-Nakanishi H, Hisabori T. Chloroplast cyclophilin is a target protein of thioredoxin. Thiol modulation 
of the peptidyl-prolyl cis-trans isomerase activity. J Biol Chem. 2003:278(34):31848-52.

27. Michelet L, Zaffagnini M, Morisse S, Sparla F, Perez-Perez ME, Francia F, et al. Redox regulation of the Calvin-Benson cycle: something old, something new. Front Plant Sci. 2013;4:470

28. Serrato AJ, Fernandez-Trijueque J, Barajas-Lopez JD, Chueca A, Sahrawy M. Plastid thioredoxins: a "one-for-all" redox-signaling system in plants. Front Plant Sci. 2013:4:463.

29. Balsera M, Uberegui E, Schurmann P, Buchanan BB. Evolutionary development of redox regulation in chloroplasts. Antioxid Redox Signal. 2014;21(9):1327-55.

30. Geigenberger P, Fernie AR. Metabolic control of redox and redox control of metabolism in plants. Antioxid Redox Signal. 2014;21(9):1389-421.

31. Li C, Wen A, Shen B, Lu J, Huang Y, Chang Y. FastCloning: a highly simplified, purification-free, sequence- and ligation-independent PCR cloning method. BMC Biotechnol. 2011;11:92.

32. Motohashi K, Kondoh A, Stumpp MT, Hisabori T. Comprehensive survey of proteins targeted by chloroplast thioredoxin. Proc Natl Acad Sci U S A. 2001:98(20):11224-9.

33. Yamaryo Y, Motohashi K, Takamiya K, Hisabori T, Ohta H. In vitro reconstitution of monogalactosyldiacylglycerol (MGDG) synthase regulation by thioredoxin. FEBS Lett. 2006;580(17):4086-90.

34. Granger JB, Lu Z, Ferguson JB, Santa Maria PJ, Novak WR. Cloning expression, purification and characterization of an iron-dependent regulator protein from Thermobifida fusca. Protein Expr Purif. 2013;92(2):190-4.

35. Wang HC, Chen YC, Huang $C T$, Hseu RS. Cloning and characterization of a thermostable and pH-stable cellobiohydrolase from Neocallimastix patriciarum J11. Protein Expr Purif. 2013;90(2):153-9.

36. Yang $Y$, Ma J, Zhang $X$, Wang $Y$, Yang L, Sun $M$. Yeast 3,'5'-bisphosphate nucleotidase: an affinity tag for protein purification. Protein Expr Purif. 2014:97:81-7.

37. Pournejati R, Karbalaei-Heidari HR, Budisa N. Secretion of recombinant archeal lipase mediated by SVP2 signal peptide in Escherichia coli and its optimization by response surface methodology. Protein Expr Purif. 2014;101:84-90.

38. Motohashi K, Romano PG, Hisabori T. Identification of thioredoxin targeted proteins using thioredoxin single cysteine mutant-immobilized resin. Methods Mol Biol. 2009;479:117-31.

39. Motohashi K, Hisabori T. CcdA is a thylakoid membrane protein required for the transfer of reducing equivalents from stroma to thylakoid lumen in the higher plant chloroplast. Antioxid Redox Signal. 2010;13(8):1169-76.

40. Motohashi K, Okegawa Y. Method for enhancement of plant redox-related protein expression and its application for in vitro reduction of chloroplastic thioredoxins. Protein Expr Purif. 2014;101:152-6.

41. Hamilton MD, Nuara AA, Gammon DB, Buller RM, Evans DH. Duplex strand joining reactions catalyzed by vaccinia virus DNA polymerase. Nucleic Acids Res. 2007;35(1):143-51.

42. Zhu B, Cai G, Hall EO, Freeman GJ. In-fusion assembly: seamless engineering of multidomain fusion proteins, modular vectors, and mutations. Biotechniques. 2007:43(3):354-9.

43. Gibson DG, Smith HO, Hutchison 3rd CA, Venter JC, Merryman C. Chemical synthesis of the mouse mitochondrial genome. Nat Methods. 2010;7(11):901-3.

44. Inoue $\mathrm{H}$, Nojima $\mathrm{H}$, Okayama $\mathrm{H}$. High efficiency transformation of Escherichia coli with plasmids. Gene. 1990;96(1):23-8.

45. Grant SG, Jessee J, Bloom FR, Hanahan D. Differential plasmid rescue from transgenic mouse DNAs into Escherichia coli methylation-restriction mutants. Proc Natl Acad Sci U S A. 1990;87(12):4645-9.

46. Yanisch-Perron C, Vieira J, Messing J. Improved M13 phage cloning vectors and host strains: nucleotide sequences of the M13mp18 and pUC19 vectors. Gene. 1985;33(1):103-19.

47. Bethesda Research Laboratories. BRL pUC host: E. coli DH5a ${ }^{T M}$ competent cells. Focus. 1986:8:9-12.

48. Dietz KJ, Horling F, Konig J, Baier M. The function of the chloroplast 2-cysteine peroxiredoxin in peroxide detoxification and its regulation J Exp Bot. 2002;53(372):1321-9.

49. Brehelin C, Meyer EH, de Souris JP, Bonnard G, Meyer Y. Resemblance and dissemblance of Arabidopsis type II peroxiredoxins: similar sequences for divergent gene expression, protein localization, and activity. Plant Physiol. 2003;132(4):2045-57.
50. Wakao S, Benning C. Genome-wide analysis of glucose-6-phosphate dehydrogenases in Arabidopsis. Plant J. 2005;41(2):243-56.

51. Takagi M, Nishioka M, Kakihara H, Kitabayashi M, Inoue H, Kawakami B, et al. Characterization of DNA polymerase from Pyrococcus sp. strain KOD1 and its application to PCR. Appl Environ Microbiol. 1997;63(11):4504-10.

52. Motohashi K, Hisabori T. HCF164 receives reducing equivalents from stromal thioredoxin across the thylakoid membrane and mediates reduction of target proteins in the thylakoid lumen. J Biol Chem. 2006;281 (46):35039-47.

\section{Submit your next manuscript to BioMed Central and take full advantage of:}

- Convenient online submission

- Thorough peer review

- No space constraints or color figure charges

- Immediate publication on acceptance

- Inclusion in PubMed, CAS, Scopus and Google Scholar

- Research which is freely available for redistribution 